\title{
Understanding GDOP minimization in GNSS Positioning: Infinite Solutions, Finite Solutions and No Solution
}

Shuqiang Xue ${ }^{1,2}$ and Yuanxi Yang ${ }^{3}$

\author{
${ }^{1}$ (School of Geological and Surveying Engineering, Chang'an University, Yanta Road, \\ $X$ ' $^{\prime}$ ' 710054, China) \\ ${ }^{2}$ (Chinese Academy of Surveying and Mapping, Beijing, 100830, China) \\ ${ }^{3}$ (National Key Laboratory for Geo-information Engineering, Xi'an Research Institute of \\ Surveying and Mapping, Xi'an, 710054, China) \\ (E-mail: yuanxi_yang@163.com)
}

\begin{abstract}
Although purely using the GNSS (Global Navigation Satellite System) users cannot obtain the theoretical GDOP minimum unless the GNSS positioning is aided by a certain number of pseudolites, discussing this problem is still meaningful in understanding the issues about the positioning geometry, such as the PDOP minimization. Many literatures have pointed that the GDOP (Geometric Dilution of Precision) minimum in 3-D positioning is the root square of $10 / n$ where $n$ is the total number of GNSS satellites or ground-based beacons with known coordinates. As the case with five known points concerned in this paper, the current knowledge indicates that the GDOP can reach the minimum the root square of 2, but our discussion shows that the GDOP minimum with five known points cannot get the theoretical minimum the root square of 2, although there are infinite positioning configurations with the lowest PDOP. Fortunately, we can find a positioning configuration with the GDOP 1.428 which is very close to the theoretical minimum 1.414. The PDOP can always reach the theoretical minimum the root square of $9 / n$, and there are infinite solutions for $n>4$. However for GDOP minimization, only when $n>5$, infinite solutions can be obtained. The configurations with the lowest GDOPs can be given by solving a set of nonlinear algebraic equations.
\end{abstract}

Key words: GDOP; PDOP; five satellites; positioning 


\section{Introduction}

The GDOP (Geometric Dilution of Precision) is a key metric in estimating the precision of model parameters before hand, by using the design matrix or the configuration of positioning in geodesy (Langley, 1999; Yang et al., 2011a; Yang et al., 2011b). It is often used to optimize the configuration (Langley, 1999; Massatt and Rudnick, 1990; Teng et al., 2015; Teunissen, 1998; Xue and Yang, 2015; Yang and Xu, 2016; Yarlagadda et al., 2000). GDOP is also important in optimally determining the configuration of ground tracking stations and in properly placing the inertial sensors (Shim and Yang, 2010; Sukkarieh, 2000; Yuksel, 2011). To optimally design a geodetic network is factually to minimize the possible GDOP which has been widely applied in terrestrial geodetic observation configuration design and satellites selection for fast positioning (Kihara and Okada, 1984; Sharp et al., 2009; Zhang and Zhang, 2009). In the case that the available observation resources is limited, the GDOP minimization of the positioning configuration should be a very crucial factor for GNSS (Global Navigation Satellite System) system design, indoor positioning and underwater positioning (Sharp et al., 2012; Zhao et al., 2015). In satellite constellation design, satellites should be treated as a series of moving known points, the dynamic configuration and best dynamic configurations have been studied (Sato et al., 2013; Xue et al., 2014b; Zhao et al., 2015). For multi-Global Navigation Satellite System (GNSS) constellations, the GDOP minimization problem is also discussed (Han et al., 2013; Teng and Wang, 2014; Teng et al., 2015).

The GDOP coverage performance around a point will be optimal if the GDOP at the point get the GDOP minimum since the GDOP is a continuous function about the unknown point and known points. Optimal configurations with a certain number of control stations, such as triangle, tetragon, regular polygon and regular tetrahedron configurations have been thoroughly studied and applied in geodetic network design (Kihara and Okada, 1984; Langley, 1999; Levanon, 2000; Phillips, 1984). A framework for analytically solving the GDOP optimization problem with arbitrary observational degrees of freedom was established by Xue and Yang (2015). In the established framework, a series of simplex configurations with the lowest GDOP, called base configurations, are given, and general configurations are created by arbitrarily combining those base configurations. These 
methods can yield a large amount of configurations with the lowest GDOP, especially with relatively high observational degrees of freedom, but they are still limited by the variety of the base configurations used.

Although the lowest GDOP configurations for 3-D positioning with four control stations and with no less six known points have been given, the case with five known points has never been solved. For the case with four satellites, it is well known that the regular tetrahedron is the only solution appeared in papers and handbooks about the GNSS positioning and navigation; for the case with six points or more points, infinite configurations with the lowest GDOP $\sqrt{10 / n}$ can be given by the Cones given by Xue and Yang (2015) where $n$ is the number of known points. The knowledge for the case with five known points is that the GDOP minimum can get $\sqrt{2}$, but there is no literature giving a positioning geometry with the GDOP $\sqrt{2}$. Does an ideal configuration with the GDOP $\sqrt{2}$ exist? In this contribution, we try to answer this question by employing the algebraic method. In Section 2, the GDOP minimization problem is introduced to briefly show the current state of knowledge of the lowest GDOP. In Section 3, the PDOP minimization is discussed and the complete solutions are given by the proposed algebraic method. An appendix A and B are given to show the complete solutions with the lowest PDOP in cases of four known points and five known points, respectively. In Section 4, the non-existence about the theoretical GDOP minimum for the case with five known points is proved by the Appendix C. A sub-optimal configuration with five points is given and the GDOP is about 1.428 .

\section{GDOPs and theoretical minimums in positioning}

\subsection{GDOPs for positioning and their minimum}

For a positioning configuration composed of $n$ points with known coordinates, the unknown coordinates can be determined by the distances or pseudo-distances from the known stations to the unknown stations with the positioning model (Xue et al., 2014a)

$$
l_{i}=d_{i}(\mathbf{x})+\Delta+\varepsilon_{i} \quad i=1 ; \cdots n
$$

where $d_{i}(\mathbf{x})=\sqrt{\left(x-x_{i}\right)^{2}+\left(y-y_{i}\right)^{2}+\left(x_{3}-z_{i}\right)^{2}}$ is the Euclidean distance from the 
unknown position $\mathbf{x}=[x, y, z] \in R^{3}$ to the $i$ th known point $\left[x_{i}, y_{i}, z_{i}\right] \in R^{3}, \varepsilon_{i}$ is the observation error with zero mean and variance $\sigma_{0}^{2}, \Delta$ is the receiver clock offset caused by the bias for pseudo-distance measurements. The positioning precision is related to two kinds of GDOPs, one of which is the PDOP defined as

$$
G D O P^{\prime}=\sqrt{\operatorname{tr}\left[\left(\mathbf{J}^{\prime \mathrm{T}} \mathbf{J}^{\prime}\right)^{-1}\right]}
$$

where

$$
\mathbf{J}^{\prime}:=\left[\begin{array}{llll}
\mathbf{e}_{1}^{\mathrm{T}} & \mathbf{e}_{2}^{\mathrm{T}} & \cdots & \mathbf{e}_{n}^{\mathrm{T}}
\end{array}\right]^{\mathrm{T}}
$$

is the Jacobin matrix of distance equations,

$$
\mathbf{e}_{i}=\left[\begin{array}{lll}
x_{i}-x & y_{i}-y & z_{i}-z
\end{array}\right] / d(\mathbf{x})
$$

is the line of sight from the unknown point to the known point; the other is defined as

$$
G D O P=\sqrt{\operatorname{tr}\left[\left(\mathbf{J}^{\mathrm{T}} \mathbf{J}\right)^{-1}\right]}
$$

where

$$
\mathbf{J}:=\left[\begin{array}{ll}
\mathbf{J}^{\prime} & \mathbf{k}_{n}
\end{array}\right]
$$

is the Jacobin matrix of pseudo-distance equations,

$$
\mathbf{k}_{n}=\left[\begin{array}{lll}
1 & \ldots & 1
\end{array}\right]^{\mathrm{T}}
$$

is the design matrix for determining the clock offset.

Based on the GDOP definition, the concepts about the PDOP (position dilution of precision), HDOP (horizontal dilution of precision), VDOP (vertical dilution of precision), TDOP (time dilution of precision) can be easily extended, and they satisfies

$$
G D O P^{2}=P D O P^{2}+T D O P^{2}=H D O P^{2}+V D O P^{2}+T D O P^{2}
$$

Current studies show that the theoretical minimums about the GDOPs are given as follows (Xue and Yang, 2015)

$$
\begin{cases}G D O P_{\min }^{\prime}=\sqrt{9 / n} & \text { if and only if } \mathbf{J}^{\prime \mathrm{T}} \mathbf{J}^{\prime}=n \mathbf{I} / 3 \\ G D O P_{\min }=\sqrt{10 / n} & \text { if and only if } \mathbf{J}^{\prime \mathrm{T}} \mathbf{J}^{\prime}=n \mathbf{I} / 3 \text { and } \mathbf{k}_{n}^{\mathrm{T}} \mathbf{J}^{\prime}=\mathbf{0}\end{cases}
$$

The above equation indicates that the GDOP' minimization condition is given by the matrix equation as follows 


$$
\mathbf{J}^{\prime \mathrm{T}} \mathbf{J}^{\prime}=n \mathbf{I} / 3
$$

while the GDOP minimization condition is given by the matrix equation as follows

$$
\left\{\begin{array}{l}
\mathbf{J}^{\mathrm{T}} \mathbf{J}^{\prime}=n \mathbf{I} / 3 \\
\mathbf{k}_{n}^{\mathrm{T}} \mathbf{J}^{\prime}=\mathbf{0}
\end{array}\right.
$$

The lowest GDOP is desired in positioning and a great number of positioning configurations with the lowest GDOP have been given, including cones, regular polyhedrons, Descartes configurations, helical configurations, Walker configurations, and their arbitrary combinations (Xue and Yang, 2015). However, for the case with five known points, no literature can give an optimal configuration with the GDOP $\sqrt{2}$. Note that:

(a) The equation (10) and (11) are both nonlinear, so there might be a unique solution, finite solutions, infinite solutions and no solution;

(b) Unconstrained case needs to be distinguished from constrained case. Some constrains might need to be introduced according to practical circumstances, such as in pure GNSS positioning the following constrains

$$
e_{k, 3} \geq 0 \quad, \quad k=1,2, \ldots, n
$$

need to be introduced where $e_{i, 3}$ is the vertical coordinate of the line of sight. Only for GNSS positioning aided with a certain number of pseudolites, the constrains (12) can be ignored;

(c) The second equation $\mathbf{k}_{n}^{\mathrm{T}} \mathbf{J}^{\prime}=\mathbf{0}$ appeared in (11) may be strong for minimizing the GDOP in practice, such as in the underwater positioning, the GNSS buoys are distributed on the sea level such that the symmetry of the positioning configuration will be very bad in the vertical direction, unless a set of known points on the seafloor are introduced to the positioning. This indicates that GDOP of a symmetrical configuration is smaller than that of an asymmetrical configuration.

In the following discussion, the discussion is based on the equation (10) and (11) without any constrains. The discussion with simple constrains can be easily taken into account, such as with the constrains (12), the number of configuration with GDOP' will be reduced by half while no configuration can get the GDOP minimum $\sqrt{10 / n}$. 


\section{Configurations with the lowest GDOP': finite solutions and infinite solutions}

Before discussing the GDOP minimization for the case with five known points, we firstly show the complete solutions for minimizing the GDOP' (PDOP as well).

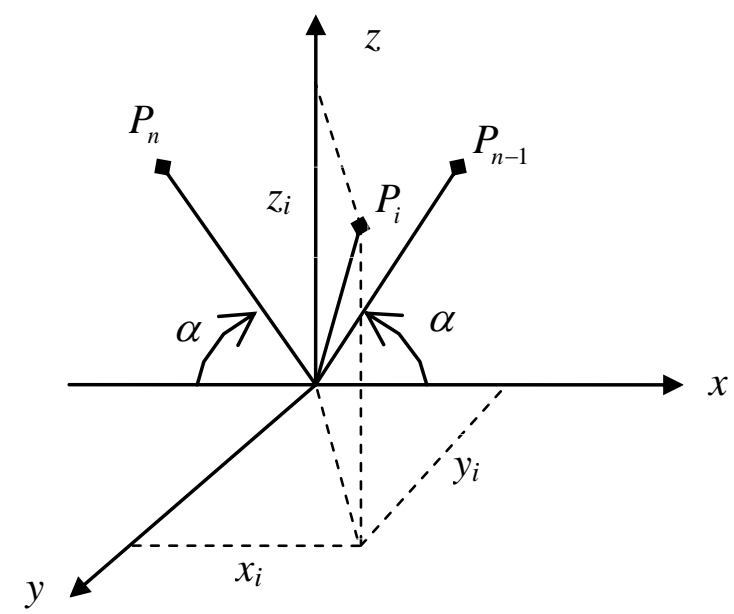

Fig. 1 Positioning configuration under the station-origin coordinate system

The GDOPs of a positioning configuration is invariant under the coordinate transformation. As shown in Fig. 1, for the convenience of discussion, the origin of coordinate system is located at the unknown point, the $z$ axis is the symmetry axis of the unknown point $P_{n}$ and $P_{n-1}$, then the Jacobin matrix (3) becomes

$$
\mathbf{J}^{\prime}=\left[\begin{array}{ccc}
x_{1} & y_{1} & z_{1} \\
x_{2} & y_{2} & z_{2} \\
\vdots & \vdots & \vdots \\
x_{n-2} & y_{n-2} & z_{n-2} \\
\cos \alpha & 0 & \sin \alpha \\
\cos \alpha & 0 & -\sin \alpha
\end{array}\right]
$$

According to the GDOP' minimization condition (10), we obtain the following algebraic equations:

$$
\left\{\begin{array}{l}
x_{k}^{2}+y_{k}^{2}+z_{k}^{2}=1, k=1,2, \ldots, n-2 \\
x_{1} y_{1}+x_{2} y_{2}+\ldots+x_{n-2} y_{n-2}=0 \\
x_{1} z_{1}+x_{2} z_{2}+\ldots+x_{n-2} z_{n-2}=0 \\
y_{1} z_{1}+y_{2} z_{2}+\ldots+y_{n-2} z_{n-2}=0 \\
y_{1}^{2}+y_{2}^{2}+\ldots+y_{n-2}^{2}=n / 3 \\
x_{1}^{2}+x_{2}^{2}+\ldots+x_{n-2}^{2}+2 \cos ^{2} \alpha=n / 3 \\
z_{1}^{2}+z_{2}^{2}+\ldots+z_{n-2}^{2}+2 \sin ^{2} \alpha=n / 3
\end{array}\right.
$$


Adding up the last three equations in (14), we have

$$
y_{1}^{2}+y_{2}^{2}+\ldots+y_{n-2}^{2}+x_{1}^{2}+x_{2}^{2}+\ldots+x_{n-2}^{2}+z_{1}^{2}+z_{2}^{2}+\ldots+z_{n-2}^{2}=n-2
$$

which can be also obtained by adding up the first $n-2$ equations in (14), so one of the last three equations in (14) can be derived by the left two equations and the first $n$ - 2 equations of (14). Therefore, although there are $n+4$ equations in (14), there are only $n+3$ independent equations. Without loss of generality, we can derive the minimum of GDOP by using the following $n+3$ equations

$$
\left\{\begin{array}{l}
x_{k}^{2}+y_{k}^{2}+z_{k}^{2}=1, k=1,2, \ldots, n-2 \\
x_{1} y_{1}+x_{2} y_{2}+\ldots+x_{n-2} y_{n-2}=0 \\
x_{1} z_{1}+x_{2} z_{2}+\ldots+x_{n-2} z_{n-2}=0 \\
y_{1} z_{1}+y_{2} z_{2}+\ldots+y_{n-2} z_{n-2}=0 \\
y_{1}^{2}+y_{2}^{2}+\ldots+y_{n-2}^{2}=n / 3 \\
x_{1}^{2}+x_{2}^{2}+\ldots+x_{n-2}^{2}+2 \cos ^{2} \alpha=n / 3
\end{array}\right.
$$

In the equation (16), there are $3 n-5$ unknowns. For $n=4$, there are 7 unknowns with 7 equations in (16), so the equation (16) have finite solutions, see the appendix A. However, because $3 n-5 \geq n+3$ for $n>4$, the unknowns in (16) is always larger than the equation numbers, so there are infinite solutions satisfying (16), e.g., for $n=5$, as shown in the Appendix B, we have 8 equations for solving 10 unknowns.

It is easy to find from (16), that the key for solving this problem is to solve the first $n+2$ equations as

$$
\left\{\begin{array}{l}
x_{k}^{2}+y_{k}^{2}+z_{k}^{2}=1, k=1,2, \ldots, n-2 \\
x_{1} y_{1}+x_{2} y_{2}+\ldots+x_{n-2} y_{n-2}=0 \\
x_{1} z_{1}+x_{2} z_{2}+\ldots+x_{n-2} z_{n-2}=0 \\
y_{1} z_{1}+y_{2} z_{2}+\ldots+y_{n-2} z_{n-2}=0 \\
y_{1}^{2}+y_{2}^{2}+\ldots+y_{n-2}^{2}=n / 3
\end{array}\right.
$$

Let the first $n+2$ parameters in $\left[x_{1}, x_{2}, \ldots, x_{n-2}, y_{1}, y_{2}, \ldots, y_{n-2}, z_{1}, z_{2}, \ldots, z_{n-2}\right]$ as unknowns while the left $2 n-8$ parameters as the freedom variables, then the solution of equation (17) can be expressed as

$$
\left[x_{1}, x_{2}, \ldots, x_{n-2}, y_{1}, y_{2}, \ldots, y_{j}, z_{1}, z_{2}, \ldots, z_{k}\right]=\mathbf{f}^{\prime}\left[y_{j+1}, y_{j+2}, \ldots, y_{n-2}, z_{j+1}, z_{j+2}, \ldots, z_{n-2}\right]
$$

where $\mathbf{f}^{\prime}$ is the explicit mapping implied by (17) which may be analytically solved, where 
$j+k=4$, and

$$
0 \leq y_{j+1}, y_{j+2}, \ldots, y_{n-2}, z_{j+1}, z_{j+2}, \ldots, z_{n-2} \leq 1
$$

For properly giving initial conditions satisfying (19), we can easily obtain one or more positioning configurations with the lowest GDOP', e.g., for $n=5$, a series of configurations with the lowest GDOP' are given in the Appendix B. Note that:

(1) Among the obtained configurations with the lowest GDOP, we can select one or more configurations with the smaller GDOP from these solutions by computing and comparing the GDOPs;

(2) The geometrical method proposed by Xue and Yang (2015) can give one or more families of solutions, but it is hard to give all families of solutions. Since the discussion in this section is based on solving the algebraic equations, so the complete solutions can be given by the method proposed above. However, the solutions with practical geometrical meanings are also needed, such as the GNSS constellation design, the GNSS orbit must obey the Kepler's equation, so the Walker constellation and the helical configuration based on the geometrical method seems more meaningful than giving infinite solutions without regard to the reality;

(3) The equation can be solved by the computer algebra software, such as the Mathematica and Maple. In the last decades, the computer algebra has been successfully applied in solving geodetic problems (AWANGE et al., 2004; Awange et al., 2010; Awange et al., 2003; Zaletnyik et al., 2008).

\section{Configurations with the lowest GDOP: unique solution, no solution and infinite solutions}

As discussed in the section 3, the GDOP' can always reach the theoretical minimum $\sqrt{9 / n}$, and there are finite solutions for $n=4$ while infinite solutions for $n \geq 5$. A new problem is that: is there one or more solution with the lowest GDOP in the family of minimal GDOP' solutions for $n=5$ ? The answer is negative and this will be proved in the following discussion.

According to (11), for discussing the GDOP minimization, the condition $\mathbf{k}_{n}^{\mathrm{T}} \mathbf{J}^{\prime}=\mathbf{0}$ needs 
to be added to the equation (16) and it becomes

$$
\left\{\begin{array}{l}
x_{k}^{2}+y_{k}^{2}+z_{k}^{2}=1, k=1,2, \ldots, n-2 \\
x_{1} y_{1}+x_{2} y_{2}+\ldots+x_{n-2} y_{n-2}=0 \\
x_{1} z_{1}+x_{2} z_{2}+\ldots+x_{n-2} z_{n-2}=0 \\
y_{1} z_{1}+y_{2} z_{2}+\ldots+y_{n-2} y_{n-2}=0 \\
y_{1}^{2}+y_{2}^{2}+y_{3}^{2}=n / 3 \\
x_{1}^{2}+x_{2}^{2}+\ldots+x_{n-2}^{2}+2 \cos ^{2} \alpha=n / 3 \\
x_{1}+x_{2}+\ldots+x_{n-2}+2 \cos \alpha=0 \\
y_{1}+y_{2}+\ldots+y_{n-2}=0 \\
z_{1}+z_{2}+\ldots+z_{n-2}=0
\end{array}\right.
$$

After eliminating the parameter $\alpha$, the following equations are obtained

$$
\left\{\begin{array}{l}
x_{k}^{2}+y_{k}^{2}+z_{k}^{2}=1, k=1,2, \ldots, n-2 \\
x_{1} y_{1}+x_{2} y_{2}+\ldots+x_{n-2} y_{n-2}=0 \\
x_{1} z_{1}+x_{2} z_{2}+\ldots+x_{n-2} z_{n-2}=0 \\
y_{1} z_{1}+y_{2} z_{2}+\ldots+y_{n-2} y_{n-2}=0 \\
y_{1}^{2}+y_{2}^{2}+y_{3}^{2}=n / 3 \\
x_{1}^{2}+x_{2}^{2}+x_{3}^{2}+\left(x_{1}+x_{2}+\ldots+x_{n-2}\right)^{2} / 2=n / 3 \\
y_{1}+y_{2}+\ldots+y_{n-2}=0 \\
z_{1}+z_{2}+\ldots+z_{n-2}=0
\end{array}\right.
$$

in which there are $n+5$ equations. When solving the equation (21) with $3 n-6$ unknowns, e.g. $n \leq 5$, because $n+5>3 n-6$ the equation (21) might be inconsistent or there might be none of solutions satisfying the equation (21), i.e., there might be no positioning configuration with the lowest GDOP given by (9). This problem can be solved in the following three cases:

(a) $n=4$. As discussed in appendix A, it shows that the definite solution is the regular tetrahedron;

(b) $n=5$. As indicated by the appendix $\mathrm{C}$, there is no configuration with the lowest GDOP $\sqrt{2}$. By search all possible combinations with 9 equations from Eq.(21), it shows that the following configuration 


$$
\mathbf{J}^{\prime}=\left[\begin{array}{ccc}
-1 / \sqrt{6} & -\sqrt{5 / 6} & 0 \\
-1 / \sqrt{6} & \sqrt{5 / 6} & 0 \\
1 & 0 & 0 \\
-1 / \sqrt{6} & 0 & -\sqrt{5 / 6} \\
-1 / \sqrt{6} & 0 & \sqrt{5 / 6}
\end{array}\right]
$$

has the smallest GDOP 1.428 which is very close to be $\sqrt{2} \approx 1.141$.

(c) $n \geq 6$. The number of equations is smaller than the unknowns such that there are infinite configurations which can get the theoretical GDOP minimum. This problem can be solved just like the method given for minimizing the DGOP' in the section 3 . The configurations with the lowest GDOP for $n \geq 6$ ever discussed in current literatures, e.g., solutions given by Xue and Yang (2015), are only particular solutions.

It should be noted that:

(a) The above discussion only means that, the minimum about the GDOP of the configuration with five stations needs to be further discussed. This contribution only gives a reference range for discussing this problem;

(b) Further discussing the GDOP minimization with five stations has theoretical meaning but not so practical, since the solution given by (22) can meet the vast majority of system design demand. The rigorous discussion for completely solving this problem should come back to the GDOP definition which is hardly analytically solved;

(c) All solutions obtained from Appendix C are the solutions of the GDOP' minimization, so all solutions given by Appendix might be also valuable for practical applications, such as indoor positioning or positioning without clock-offset parameter;

(d) The final result (22) has the same form with result given by Teng et al. (2015) as

$$
\mathbf{J}^{\prime}=\mathbf{J}^{\prime}=\left[\begin{array}{ccc}
\cos \alpha & -\sin \alpha & 0 \\
\cos \alpha & \sin \alpha & 0 \\
1 & 0 & 0 \\
\cos \alpha & 0 & -\sin \alpha \\
\cos \alpha & 0 & \sin \alpha
\end{array}\right]
$$

which indicates that the theory base for discussing the GDOP minimization of single constellation and multi-constellation is unified. The difference is from the second equation in (11) about the clock-offset parameter's identification, so that the GDOP minimization for multi-constellation has more equations than that for single-constellation. 


\section{Conclusions}

The GDOP minimum cannot reach $\sqrt{2}$ in $3-\mathrm{D}$ positioning with five known points. Exception for the case of five control stations, the GDOP minimum can always reach $\sqrt{10 / n}$ where $n$ is the number of known points. This indicates that the current studies about GDOP minimization for $n=5$ is incomplete and this problem needs to be further discussed to give a practical minimum. However in practice this incompleteness is not a serious problem, and we have found a configuration with the small GDOP about 1.428 which is very close to be the nominal lowest GDOP 1.414 .

The PDOP can always reach the theoretical minimum $\sqrt{9 / n}$, and there are infinite solutions for $n \geq 5$. However for GDOP minimization, only when $n \geq 6$, infinite solutions can be obtained. The configurations with the lowest GDOPs can be given by solving a set of nonlinear algebraic equations, and the analytical solution can be obtained by Mathematica software by properly giving a set of initial conditions. 


\section{Appendix}

To save the space, the following Appendixes only give a hint for deriving the final solutions. The readers who want to check the derivations can run the Mathematica code given in the Appendixes.

\section{Appendix A: The lowest GDOP' Configurations with four known points}

\section{(a) Basic equations}

According to (17), for $n=4$ the following equations needs to be solved:

$$
\left\{\begin{array}{l}
x_{1}^{2}+y_{1}^{2}+z_{1}^{2}=1 \\
x_{2}^{2}+y_{2}^{2}+z_{2}^{2}=1 \\
x_{1} y_{1}+x_{2} y_{2}=0 \\
x_{1} z_{1}+x_{2} z_{2}=0 \\
y_{1} z_{1}+y_{2} z_{2}=0 \\
y_{1}^{2}+y_{2}^{2}=4 / 3
\end{array}\right.
$$

\section{(b) Mathematica codes:}

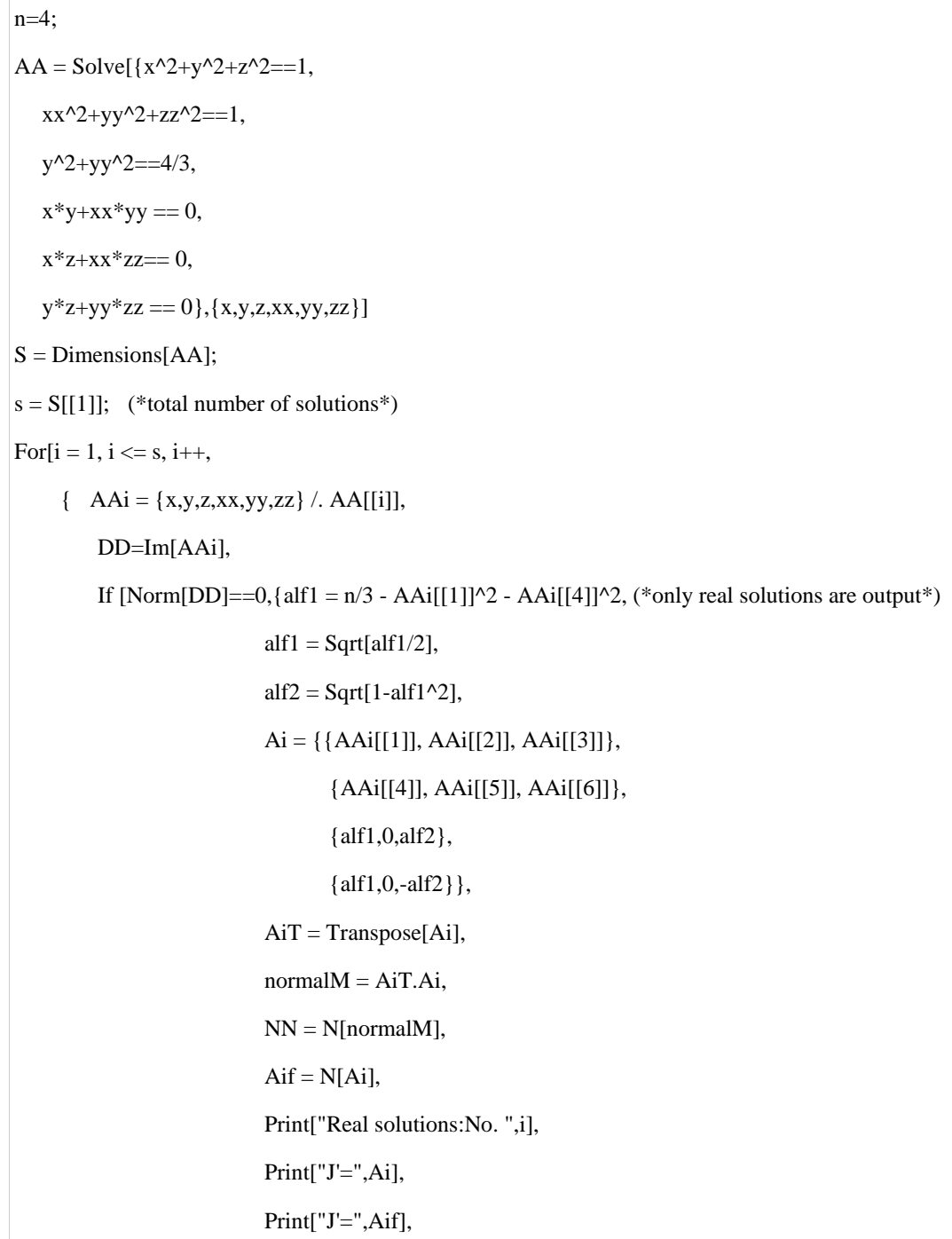


$\operatorname{Print}\left[" \mathrm{~J}^{\prime} * \mathrm{~J}^{\prime}=", \mathrm{NN}\right]$,

GDOP $=\operatorname{Sqr}[\operatorname{Tr}[$ Inverse[NN]],

Print["GDOP'=",GDOP]\}

]

(c) There are 16 outputs but there are only four different solutions which can be also obtained by the following manual derivations

\section{(d) Manual derivations:}

TBy using the orthogonality condition given by the third, fourth and fifth equations in (24), for $z_{1}, z_{2} \neq 0$ we have the following derivations:

$$
\left.\begin{array}{l}
\frac{x_{1}}{x_{2}}=-\frac{y_{2}}{y_{1}} \\
\frac{x_{1}}{x_{2}}=-\frac{z_{2}}{z_{1}} \\
-\frac{y_{2}}{y_{1}}=\frac{z_{1}}{z_{2}}
\end{array}\right\} \Rightarrow-\frac{z_{2}}{z_{1}}=\frac{z_{1}}{z_{2}} \Rightarrow z_{1}=z_{2}=0
$$

This shows that $z_{1}=z_{2}=0$, then the equation (24) becomes

$$
\left\{\begin{array}{l}
x_{1}^{2}+y_{1}^{2}=1 \\
x_{2}^{2}+y_{2}^{2}=1 \\
x_{1} y_{1}+x_{2} y_{2}=0 \\
y_{1}^{2}+y_{2}^{2}=\frac{4}{3}
\end{array}\right.
$$

With the third equation in (26), let $x_{1}=k x_{2}, y_{2}=-k y_{1}$, we have

$$
\left.\begin{array}{l}
k^{2} x_{2}^{2}+y_{1}^{2}=1 \\
x_{2}^{2}+k^{2} y_{1}^{2}=1 \\
y_{1}^{2}+k^{2} y_{1}^{2}=\frac{4}{3}
\end{array}\right\}
$$

Sloving the equation (27), we have $k^{2}=1$ and

$$
\left\{\begin{array}{l}
y_{1}= \pm \sqrt{\frac{2}{3}} \\
x_{2}= \pm \sqrt{\frac{1}{3}}
\end{array}\right.
$$


The left unknowns can be solved by performing the back substitution.

Note that, the software gives 16 solutions, but in fact they only gives four difference configurations including the regular tetrahedron and its reflections, about the reflection operation, see the results given by Xue et. al (2015).

\section{Appendix B: The lowest GDOP' Configurations with five known points}

\section{(a) Basic equations}

According to (17), for $n=5$ the following equations needs to be solved:

$$
\left\{\begin{array}{l}
x_{1}^{2}+y_{1}^{2}+z_{1}^{2}=1 \\
x_{2}^{2}+y_{2}^{2}+z_{2}^{2}=1 \\
x_{3}^{2}+y_{3}^{2}+z_{3}^{2}=1 \\
x_{1} y_{1}+x_{2} y_{2}+x_{3} y_{3}=0 \\
x_{1} z_{1}+x_{2} z_{2}+x_{3} z_{3}=0 \\
y_{1} z_{1}+y_{2} z_{2}+y_{3} z_{3}=0 \\
y_{1}^{2}+y_{2}^{2}+y_{3}^{2}=\frac{5}{3}
\end{array}\right.
$$

\section{(b) Mathematica codes:}

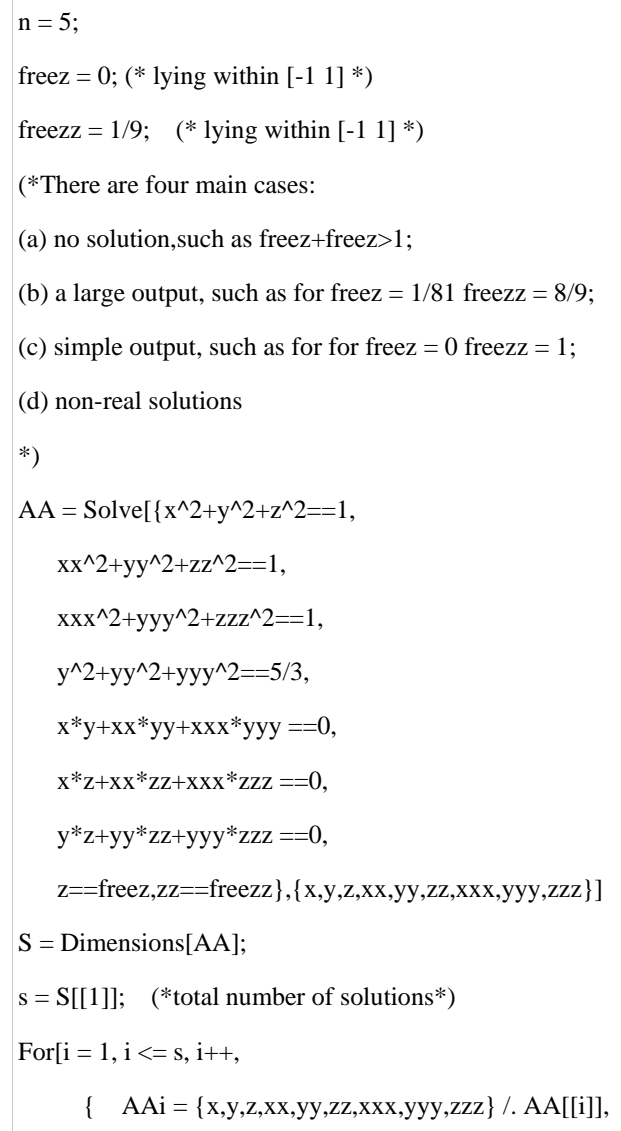


$\mathrm{DD}=\operatorname{Im}[\mathrm{AAi}]$

If $\left[\operatorname{Norm}[\mathrm{DD}]==0,\left\{\right.\right.$ alf1 $=\mathrm{n} / 3-\mathrm{AAi}[[1]]^{\wedge} 2-\mathrm{AAi}[[4]]^{\wedge} 2-\mathrm{AAi}[[7]]^{\wedge} 2,\left(*\right.$ only real solutions are output $\left.{ }^{*}\right)$

alf1 $=$ Sqrt[alf1/2],

alf $2=\operatorname{Sqrt}\left[1-\operatorname{alf} 1^{\wedge} 2\right]$,

$\mathrm{Ai}=\{\{\operatorname{AAi}[[1]], \operatorname{AAi}[[2]], \operatorname{AAi}[[3]]\}$,

$\{$ AAi[[4]], AAi[[5]], AAi[[6]]\},

\{AAi[[7]], AAi[[8]], AAi[[9]]\},

$\{$ alf1,0,alf2 $\}$,

$\{$ alf1,0,-alf 2$\}$,

$\mathrm{AiT}=$ Transpose[Ai]

normalM = AiT.Ai,

$\mathrm{NN}=\mathrm{N}[$ normalM $]$

Aif $=\mathrm{N}[\mathrm{Ai}]$,

Print["Real solutions:No. ",i],

$\operatorname{Print}[" J '=", A i]$,

Print["J'=",Aif],

$\operatorname{Print}\left[" \mathrm{~J}^{\prime} \mathrm{J}^{\prime}=", \mathrm{NN}\right]$,

GDOP $=\operatorname{Sqrt}[\operatorname{Tr}[\operatorname{Inverse}[\mathrm{NN}]]]$,

Print["GDOP'=",GDOP]\}

]

(c) Real solutions from the Mathematica software (for $z_{1}=0, z_{2}=1 / 9$ ):

$$
\begin{aligned}
& \left\{x \rightarrow \frac{1}{\sqrt{6}}, y \rightarrow-\sqrt{\frac{5}{6}}, z \rightarrow 0, \mathrm{xx} \rightarrow-\frac{2 \sqrt{\frac{10}{3}}}{9}, \mathrm{yy} \rightarrow-\frac{10 \sqrt{\frac{2}{3}}}{9}, \mathrm{zz} \rightarrow \frac{1}{9}, \mathrm{xxx} \rightarrow-\frac{1}{9 \sqrt{6}}, \mathrm{yyy} \rightarrow-\frac{\sqrt{\frac{5}{6}}}{9}, \mathrm{zzz} \rightarrow-\frac{4 \sqrt{5}}{9}\right\}, \\
& \left\{x \rightarrow-\frac{1}{\sqrt{6}}, y \rightarrow-\sqrt{\frac{5}{6}}, z \rightarrow 0, \mathrm{xx} \rightarrow \frac{2 \sqrt{\frac{10}{3}}}{9}, \mathrm{yy} \rightarrow-\frac{10 \sqrt{\frac{2}{3}}}{9}, \mathrm{zz} \rightarrow \frac{1}{9}, \mathrm{xxx} \rightarrow \frac{1}{9 \sqrt{6}}, \mathrm{yyy} \rightarrow-\frac{\sqrt{\frac{5}{6}}}{9}, \mathrm{zzz} \rightarrow-\frac{4 \sqrt{5}}{9}\right\}, \\
& \left\{x \rightarrow-\frac{1}{\sqrt{6}}, y \rightarrow \sqrt{\frac{5}{6}}, z \rightarrow 0, \mathrm{xx} \rightarrow-\frac{2 \sqrt{\frac{10}{3}}}{9}, \mathrm{yy} \rightarrow-\frac{10 \sqrt{\frac{2}{3}}}{9}, \mathrm{zz} \rightarrow \frac{1}{9}, \mathrm{xxx} \rightarrow-\frac{1}{9 \sqrt{6}}, \mathrm{yyy} \rightarrow-\frac{\sqrt{\frac{5}{6}}}{9}, \mathrm{zzz} \rightarrow-\frac{4 \sqrt{5}}{9}\right\}, \\
& \left\{x \rightarrow \frac{1}{\sqrt{6}}, y \rightarrow \sqrt{\frac{5}{6}}, z \rightarrow 0, \mathrm{xx} \rightarrow \frac{2 \sqrt{\frac{10}{3}}}{9}, \mathrm{yy} \rightarrow-\frac{10 \sqrt{\frac{2}{3}}}{9}, \mathrm{zz} \rightarrow \frac{1}{9}, \mathrm{xxx} \rightarrow \frac{1}{9 \sqrt{6}}, \mathrm{yyy} \rightarrow-\frac{\sqrt{\frac{5}{6}}}{9}, \mathrm{zzz} \rightarrow-\frac{4 \sqrt{5}}{9}\right\}, \\
& \left\{x \rightarrow \frac{1}{\sqrt{6}}, y \rightarrow-\sqrt{\frac{5}{6}}, z \rightarrow 0, \mathrm{xx} \rightarrow \frac{2 \sqrt{\frac{10}{3}}}{9}, \mathrm{yy} \rightarrow \frac{10 \sqrt{\frac{2}{3}}}{9}, \mathrm{zz} \rightarrow \frac{1}{9}, \mathrm{xxx} \rightarrow-\frac{1}{9 \sqrt{6}}, \mathrm{yyy} \rightarrow-\frac{\sqrt{\frac{5}{6}}}{9}, \mathrm{zzz} \rightarrow \frac{4 \sqrt{5}}{9}\right\}, \\
& \left\{x \rightarrow-\frac{1}{\sqrt{6}}, y \rightarrow-\sqrt{\frac{5}{6}}, z \rightarrow 0, \mathrm{xx} \rightarrow-\frac{2 \sqrt{\frac{10}{3}}}{9}, \mathrm{yy} \rightarrow \frac{10 \sqrt{\frac{2}{3}}}{9}, \mathrm{zz} \rightarrow \frac{1}{9}, \mathrm{xxx} \rightarrow \frac{1}{9 \sqrt{6}}, \mathrm{yyy} \rightarrow-\frac{\sqrt{\frac{5}{6}}}{9}, \mathrm{zzz} \rightarrow \frac{4 \sqrt{5}}{9}\right\}, \\
& \left\{x \rightarrow-\frac{1}{\sqrt{6}}, y \rightarrow \sqrt{\frac{5}{6}}, z \rightarrow 0, \mathrm{xx} \rightarrow \frac{2 \sqrt{\frac{10}{3}}}{9}, \mathrm{yy} \rightarrow \frac{10 \sqrt{\frac{2}{3}}}{9}, \mathrm{zz} \rightarrow \frac{1}{9}, \mathrm{xxx} \rightarrow-\frac{1}{9 \sqrt{6}}, \mathrm{yyy} \rightarrow-\frac{\sqrt{\frac{5}{6}}}{9}, \mathrm{zzz} \rightarrow \frac{4 \sqrt{5}}{9}\right\},
\end{aligned}
$$




$$
\begin{aligned}
& \left\{\mathrm{x} \rightarrow \frac{1}{\sqrt{6}}, \mathrm{y} \rightarrow \sqrt{\frac{5}{6}}, \mathrm{z} \rightarrow 0, \mathrm{xx} \rightarrow-\frac{2 \sqrt{\frac{10}{3}}}{9}, \mathrm{yy} \rightarrow \frac{10 \sqrt{\frac{2}{3}}}{9}, \mathrm{zz} \rightarrow \frac{1}{9}, \mathrm{xxx} \rightarrow \frac{1}{9 \sqrt{6}}, \mathrm{yyy} \rightarrow-\frac{\sqrt{\frac{5}{6}}}{9}, \mathrm{zzz} \rightarrow \frac{4 \sqrt{5}}{9}\right\}, \\
& \left\{\mathrm{x} \rightarrow-\frac{1}{\sqrt{6}}, \mathrm{y} \rightarrow-\sqrt{\frac{5}{6}}, \mathrm{z} \rightarrow 0, \mathrm{xx} \rightarrow \frac{2 \sqrt{\frac{10}{3}}}{9}, \mathrm{yy} \rightarrow-\frac{10 \sqrt{\frac{2}{3}}}{9}, \mathrm{zz} \rightarrow \frac{1}{9}, \mathrm{xxx} \rightarrow-\frac{1}{9 \sqrt{6}}, \mathrm{yyy} \rightarrow \frac{\sqrt{\frac{5}{6}}}{9}, \mathrm{zzz} \rightarrow \frac{4 \sqrt{5}}{9}\right\}, \\
& \left\{\mathrm{x} \rightarrow \frac{1}{\sqrt{6}}, \mathrm{y} \rightarrow-\sqrt{\frac{5}{6}}, \mathrm{z} \rightarrow 0, \mathrm{xx} \rightarrow-\frac{2 \sqrt{\frac{10}{3}}}{9}, \mathrm{yy} \rightarrow-\frac{10 \sqrt{\frac{2}{3}}}{9}, \mathrm{zz} \rightarrow \frac{1}{9}, \mathrm{xxx} \rightarrow \frac{1}{9 \sqrt{6}}, \mathrm{yyy} \rightarrow \frac{\sqrt{\frac{5}{6}}}{9}, \mathrm{zzz} \rightarrow \frac{4 \sqrt{5}}{9}\right\}, \\
& \left\{\mathrm{x} \rightarrow \frac{1}{\sqrt{6}}, \mathrm{y} \rightarrow \sqrt{\frac{5}{6}}, \mathrm{z} \rightarrow 0, \mathrm{xx} \rightarrow \frac{2 \sqrt{\frac{10}{3}}}{9}, \mathrm{yy} \rightarrow-\frac{10 \sqrt{\frac{2}{3}}}{9}, \mathrm{zz} \rightarrow \frac{1}{9}, \mathrm{xxx} \rightarrow-\frac{1}{9 \sqrt{6}}, \mathrm{yyy} \rightarrow \frac{\sqrt{\frac{5}{6}}}{9}, \mathrm{zzz} \rightarrow \frac{4 \sqrt{5}}{9}\right\}, \\
& \left\{\mathrm{x} \rightarrow-\frac{1}{\sqrt{6}}, \mathrm{y} \rightarrow \sqrt{\frac{5}{6}}, \mathrm{z} \rightarrow 0, \mathrm{xx} \rightarrow-\frac{2 \sqrt{\frac{10}{3}}}{9}, \mathrm{yy} \rightarrow-\frac{10 \sqrt{\frac{2}{3}}}{9}, \mathrm{zz} \rightarrow \frac{1}{9}, \mathrm{xxx} \rightarrow \frac{1}{9 \sqrt{6}}, \mathrm{yyy} \rightarrow \frac{\sqrt{\frac{5}{6}}}{9}, \mathrm{zzz} \rightarrow \frac{4 \sqrt{5}}{9}\right\}, \\
& \left\{\mathrm{x} \rightarrow-\frac{1}{\sqrt{6}}, \mathrm{y} \rightarrow-\sqrt{\frac{5}{6}}, \mathrm{z} \rightarrow 0, \mathrm{xx} \rightarrow-\frac{2 \sqrt{\frac{10}{3}}}{9}, \mathrm{yy} \rightarrow \frac{10 \sqrt{\frac{2}{3}}}{9}, \mathrm{zz} \rightarrow \frac{1}{9}, \mathrm{xxx} \rightarrow-\frac{1}{9 \sqrt{6}}, \mathrm{yyy} \rightarrow \frac{\sqrt{\frac{5}{6}}}{9}, \mathrm{zzz} \rightarrow-\frac{4 \sqrt{5}}{9}\right\}, \\
& \left\{\mathrm{x} \rightarrow \frac{1}{\sqrt{6}}, \mathrm{y} \rightarrow-\sqrt{\frac{5}{6}}, \mathrm{z} \rightarrow 0, \mathrm{xx} \rightarrow \frac{2 \sqrt{\frac{10}{3}}}{9}, \mathrm{yy} \rightarrow \frac{10 \sqrt{\frac{2}{3}}}{9}, \mathrm{zz} \rightarrow \frac{1}{9}, \mathrm{xxx} \rightarrow \frac{1}{9 \sqrt{6}}, \mathrm{yyy} \rightarrow \frac{\sqrt{\frac{5}{6}}}{9}, \mathrm{zzz} \rightarrow-\frac{4 \sqrt{5}}{9}\right\}, \\
& \left\{\mathrm{x} \rightarrow \frac{1}{\sqrt{6}}, \mathrm{y} \rightarrow \sqrt{\frac{5}{6}}, \mathrm{z} \rightarrow 0, \mathrm{xx} \rightarrow-\frac{2 \sqrt{\frac{10}{3}}}{9}, \mathrm{yy} \rightarrow \frac{10 \sqrt{\frac{2}{3}}}{9}, \mathrm{zz} \rightarrow \frac{1}{9}, \mathrm{xxx} \rightarrow-\frac{1}{9 \sqrt{6}}, \mathrm{yyy} \rightarrow \frac{\sqrt{\frac{5}{6}}}{9}, \mathrm{zzz} \rightarrow-\frac{4 \sqrt{5}}{9}\right\}, \\
& \left.\left\{\mathrm{x} \rightarrow-\frac{1}{\sqrt{6}}, \mathrm{y} \rightarrow \sqrt{\frac{5}{6}}, \mathrm{z} \rightarrow 0, \mathrm{xx} \rightarrow \frac{2 \sqrt{\frac{10}{3}}}{9}, \mathrm{yy} \rightarrow \frac{10 \sqrt{\frac{2}{3}}}{9}, \mathrm{zz} \rightarrow \frac{1}{9}, \mathrm{xxx} \rightarrow \frac{1}{9 \sqrt{6}}, \mathrm{yyy} \rightarrow \frac{\sqrt{\frac{5}{6}}}{9}, \mathrm{zzz} \rightarrow-\frac{4 \sqrt{5}}{9}\right\}\right\}
\end{aligned}
$$

\section{(d) Manual derivations:}

For arbitrary $z$ and $z z$ lying within [-1 1$]$, there may a series number of configurations with the lowest GDOP'. A general solution expression as

$$
\left[x_{1}, x_{2}, x_{3}, y_{1}, y_{2}, y_{3}, z_{1}\right]=\mathbf{f}_{5}^{\prime}\left(z_{1}, z_{2}\right)
$$

is hard to be given, so the software solution is recommended to be used in practice after properly given the initial conditions according to practical demands.

\section{Appendix C: GDOP of a configuration with five known points is always bigger than} $\sqrt{2}$

\section{(a) Manual poof}

The key for proving this property about the GDOP is to show that the equation (21) is inconsistent. For this, we can first solve the following equations of which the number of unknowns is equal to the number of equations. 


$$
\left\{\begin{array}{l}
x_{1}^{2}+y_{1}^{2}+z_{1}^{2}=1 \\
x_{2}^{2}+y_{2}^{2}+z_{2}^{2}=1 \\
x_{3}^{2}+y_{3}^{2}+z_{3}^{2}=1 \\
y_{1}^{2}+y_{2}^{2}+y_{3}^{2}=5 / 3 \\
y_{1}+y_{2}+y_{3}=0 \\
z_{1}+z_{2}+z_{3}=0 \\
x_{1} y_{1}+x_{2} y_{2}+x_{3} y_{3}=0 \\
x_{1} z_{1}+x_{2} z_{2}+x_{3} z_{3}=0 \\
y_{1} z_{1}+y_{2} z_{2}+y_{3} z_{3}=0
\end{array}\right.
$$

Eliminating the 5th and 6th equation, we can obtain:

$$
\left\{\begin{array}{l}
x_{1}^{2}+y_{2}^{2}+y_{3}^{2}+2 y_{2} y_{3}+z_{2}^{2}+z_{3}^{2}+2 z_{2} z_{3}=1 \\
x_{2}^{2}+y_{2}^{2}+z_{2}^{2}=1 \\
x_{3}^{2}+y_{3}^{2}+z_{3}^{2}=1 \\
y_{2}^{2}+y_{3}^{2}+y_{2} y_{3}=5 / 6 \\
-x_{1}\left(y_{2}+y_{3}\right)+x_{2} y_{2}+x_{3} y_{3}=0 \\
-x_{1}\left(z_{2}+z_{3}\right)+x_{2} z_{2}+x_{3} z_{3}=0 \\
\left(y_{2}+y_{3}\right)\left(z_{2}+z_{3}\right)+y_{2} z_{2}+y_{3} z_{3}=0
\end{array}\right.
$$

*Sub derivation C1.: $\left(x_{2}-x_{1}\right) \neq 0$

By the 5th and 6th equations in (32), when $\left(x_{2}-x_{1}\right) \neq 0$ we have

$$
\left\{\begin{array}{l}
y_{2}=\frac{\left(x_{1}-x_{3}\right) y_{3}}{\left(x_{2}-x_{1}\right)} \\
z_{2}=\frac{\left(x_{1}-x_{3}\right) z_{3}}{\left(x_{2}-x_{1}\right)}
\end{array}\right.
$$

Then from (33), eliminating $y_{2}$ and $z_{2}$ in (32), we can obtain

$$
\left\{\begin{array}{l}
\frac{\left(x_{1}-x_{3}\right)^{2} y_{3}^{2}}{\left(x_{2}-x_{1}\right)^{2}}+y_{3}^{2}+\frac{\left(x_{1}-x_{3}\right) y_{3}^{2}}{\left(x_{2}-x_{1}\right)}=\frac{5}{6} \\
\frac{\left(x_{1}-x_{3}\right) y_{3}}{\left(x_{2}-x_{1}\right)}\left(2 \frac{\left(x_{1}-x_{3}\right) z_{3}}{\left(x_{2}-x_{1}\right)}+z_{3}\right)+y_{3}\left(\frac{\left(x_{1}-x_{3}\right) z_{3}}{\left(x_{2}-x_{1}\right)}+2 z_{3}\right)=0 \\
\frac{\left(x_{1}-x_{3}\right) y_{3}}{\left(x_{2}-x_{1}\right)}\left(2 \frac{\left(x_{1}-x_{3}\right) z_{3}}{\left(x_{2}-x_{1}\right)}+z_{3}\right)+y_{3}\left(\frac{\left(x_{1}-x_{3}\right) z_{3}}{\left(x_{2}-x_{1}\right)}+2 z_{3}\right)=0
\end{array}\right.
$$

From the second equation in (34), we can obtain 


$$
\left\{\begin{array}{l}
y_{3} \neq 0 \\
\left(x_{1}-x_{3}\right)^{2}+\left(x_{2}-x_{1}\right)\left(x_{1}-x_{3}\right)+\left(x_{2}-x_{1}\right)^{2} \neq 0
\end{array}\right.
$$

However, from the third equation in (34), we have

$$
\left[\frac{\left(x_{1}-x_{3}\right)}{\left(x_{2}-x_{1}\right)}\left(2 \frac{\left(x_{1}-x_{3}\right)}{\left(x_{2}-x_{1}\right)}+1\right)+\left(\frac{\left(x_{1}-x_{3}\right)}{\left(x_{2}-x_{1}\right)}+2\right)\right] y_{3} z_{3}=0
$$

i.e.,

$$
\left\{\begin{array}{l}
z_{3}=0 \\
\text { or } y_{3}=0 \\
\text { or }\left(x_{1}-x_{3}\right)^{2}+\left(x_{2}-x_{1}\right)\left(x_{1}-x_{3}\right)+\left(x_{2}-x_{1}\right)^{2}=0
\end{array}\right.
$$

Comparing (36) with (35), we have $z_{3}=0$, in the same way, we can also obtain $z_{1}=0, z_{2}=0$, i.e., we have

$$
\left\{\begin{array}{l}
z_{1}=0 \\
z_{2}=0 \\
z_{3}=0
\end{array}\right.
$$

By substituting (38) into the original equation and elimating the variables $x_{1}, x_{2}, x_{3}$ by using the fist three equations, we have the following derivations:

$$
\left\{\begin{array}{l}
y_{1}^{2}+y_{2}^{2}+y_{3}^{2}=\frac{5}{3} \\
y_{1}+y_{2}+y_{3}=0 \\
\sqrt{1-y_{1}^{2}} y_{1} \pm \sqrt{1-y_{2}^{2}} y_{2} \pm \sqrt{1-y_{3}^{2}} y_{3}=0
\end{array}\right.
$$

Solving the first two equations in (39) we have

$$
\left\{\begin{array}{l}
y_{2}=-\frac{1}{2} y_{3} \pm \frac{1}{6} \sqrt{30-27 y_{3}^{2}} \\
y_{1}=-\frac{1}{2} y_{3} \mp \frac{1}{6} \sqrt{30-27 y_{3}^{2}}
\end{array}\right.
$$

With (40) we have

$$
\left\{\begin{array}{l}
1-y_{2}^{2}=\frac{1}{6}+\frac{1}{2} y_{3}^{2} \mp \frac{y_{3}}{6} \sqrt{30-27 y_{3}^{2}}=\frac{1}{6}-y_{3}\left(-\frac{1}{2} y_{3} \pm \frac{1}{6} \sqrt{30-27 y_{3}^{2}}\right)=\frac{1}{6}-y_{3} y_{2} \\
1-y_{1}^{2}=\frac{1}{6}+\frac{1}{2} y_{3}^{2} \pm \frac{y_{3}}{6} \sqrt{30-27 y_{3}^{2}}=\frac{1}{6}-y_{3}\left(-\frac{1}{2} y_{3} \mp \frac{1}{6} \sqrt{30-27 y_{3}^{2}}\right)=\frac{1}{6}-y_{3} y_{1}
\end{array}\right.
$$

and then we can obtain 


$$
y_{1}^{2}-y_{2}^{2}=y_{3} y_{1}-y_{3} y_{2}=y_{3}\left(y_{1}-y_{2}\right)
$$

Solving the equation (42) we have

$$
\begin{cases}y_{3}=0 & \text { for }\left(y_{1}-y_{2}\right) \neq 0 \\ y_{1}=y_{2} & \text { for }\left(y_{1}-y_{2}\right)=0\end{cases}
$$

Note that the first solution in (43) is obtained by combining $y_{1}+y_{2}=y_{3}$ with (40)

$* *$ Sub derivation C1.1: $y_{3}=0$

With $y_{3}=0$, coming back the equation (39) we have the following derivations

$$
\left\{\begin{array} { l } 
{ y _ { 1 } ^ { 2 } + y _ { 2 } ^ { 2 } = \frac { 5 } { 3 } } \\
{ y _ { 1 } + y _ { 2 } = 0 }
\end{array} \Rightarrow \left\{\begin{array}{l}
y_{2}= \pm \sqrt{5 / 6} \\
y_{1}=\mp \sqrt{5 / 6}
\end{array}\right.\right.
$$

By the back substitution, the solutions (44) can be used to obtain a series of real number solutions.

$$
* * \text { Sub derivation C1.2: } y_{1}=y_{2}
$$

With $y_{1}=y_{2}$, coming back the equation (39) we have the following derivations

$$
\left.\begin{array}{l}
2 y_{2}^{2}+y_{3}^{2}=\frac{5}{3} \\
2 y_{2}+y_{3}=0
\end{array}\right\} \Rightarrow y_{3}^{2}=\frac{5}{18} ; y_{2}^{2}=\frac{25}{36} ; \quad y_{1}^{2}=\frac{25}{36}
$$

By the back substitution, the solutions (45) cannot be used to obtain a series of real solutions.

\section{*Sub derivation C2.: $\left(x_{2}-x_{1}\right)=0$}

By the 5th and 6th equations in (32), when $\left(x_{2}-x_{1}\right)=0$ we have, we have

$$
\left\{\begin{array}{l}
\left(x_{3}-x_{1}\right) y_{3}=0 \\
\left(x_{3}-x_{1}\right) z_{3}=0
\end{array}\right.
$$

**Sub derivation C2.1.: $\left(x_{3}-x_{1}\right)=0$

In this case, with (46) we have

$$
x_{1}=x_{2}=x_{3} \neq 0
$$

and then elmiating the variables $x_{1}, x_{2}, x_{3}$ in (31) we have 


$$
\left\{\begin{array}{l}
y_{2}^{2}+z_{2}^{2}=y_{1}^{2}+z_{1}^{2} \\
y_{3}^{2}+z_{3}^{2}=y_{1}^{2}+z_{1}^{2} \\
y_{1}^{2}+y_{2}^{2}+y_{3}^{2}=\frac{5}{3} \\
y_{1}+y_{2}+y_{3}=0 \\
y_{1} z_{1}+y_{2} z_{2}+y_{3} z_{3}=0 \\
z_{1}+z_{2}+z_{3}=0
\end{array}\right.
$$

Further eliminting the variables $y_{1}, z_{1}$ in the above equation we can obatin

$$
\left\{\begin{array}{l}
y_{3}=-\frac{\left(z_{3}+2 z_{2}\right) z_{3}}{\left(y_{3}+2 y_{2}\right)} \\
y_{2}=-\frac{\left(z_{2}+2 z_{3}\right) z_{2}}{\left(y_{2}+2 y_{3}\right)} \\
\frac{z_{2}}{z_{3}}=-\frac{\left(y_{2}+2 y_{3}\right)}{\left(2 y_{2}+y_{3}\right)} \\
y_{2}^{2}+y_{3}^{2}+y_{2} y_{3}=5 / 6
\end{array}\right.
$$

Let $k_{3}:=z_{2} / z_{3}=-\left(y_{2}+2 y_{3}\right) /\left(2 y_{2}+y_{3}\right)$ the above equation becomes

$$
\left\{\begin{array}{l}
y_{3}=-\frac{\left(1+2 k_{3}\right) z_{3}^{2}}{\left(y_{3}+2 y_{2}\right)} \\
y_{2}=-\frac{k_{3}\left(k_{3}+2\right) z_{3}^{2}}{\left(y_{2}+2 y_{3}\right)} \\
y_{2}^{2}+y_{3}^{2}+y_{2} y_{3}=5 / 6
\end{array}\right.
$$

$* * *$ Sub derivation C2.1.1.: $y_{2} \neq 0$

With $y_{2} \neq 0$ and $k_{3}=-\left(y_{2}+2 y_{3}\right) /\left(2 y_{2}+y_{3}\right)$, by the first two equations in (50) we have

$$
\frac{y_{3}}{y_{2}}=-\frac{\left(1+2 k_{3}\right)}{\left(k_{3}+2\right)}
$$

However, with regard to $k_{3}=-\left(y_{2}+2 y_{3}\right) /\left(2 y_{2}+y_{3}\right)$ we always have the realtions as

$$
\frac{y_{3}}{y_{2}}=-\frac{\left(4+2 k_{3}\right)-3}{\left(k_{3}+2\right)}=\frac{3}{\left(k_{3}+2\right)}-2 \frac{3}{2-\left(y_{2}+2 y_{3}\right) /\left(2 y_{2}+y_{3}\right)}-2=\frac{\left(2 y_{2}+y_{3}\right)}{y_{2}}-2=\frac{y_{3}}{y_{2}}
$$

The above derivations indicate that, in this case, the original equation has infinite solutions, and the infinite solutions can be expressed as a function about the variable $y_{3}$, 
e.g., $\quad y_{2}$ can be expressed as

$$
y_{2}^{2}+y_{3}^{2}+y_{2} y_{3}=\frac{5}{6} \Rightarrow y_{2}=-\frac{1}{2} y_{3} \pm \frac{1}{6} \sqrt{30-27 y_{3}^{2}}
$$

By the back substitution, we can obtain the final solutions, but the solutions are not real solutions.

\section{$* * *$ Sub derivation C2.1.2.: $y_{2}=0$}

Substituting $y_{2}=0$ into the equation (48), we can obtain

$$
\left\{\begin{array}{l}
z_{2}^{2}=y_{1}^{2}+z_{1}^{2} \\
y_{3}^{2}+z_{3}^{2}=y_{1}^{2}+z_{1}^{2} \\
y_{1}^{2}+y_{3}^{2}=5 / 3 \\
y_{1}+y_{3}=0 \\
y_{1} z_{1}+y_{3} z_{3}=0 \\
z_{1}+z_{2}+z_{3}=0
\end{array}\right.
$$

and then

$$
z_{2}^{2}=5 / 18 ; y_{1}^{2}=5 / 6 ; y_{3}^{2}=5 / 6 ; y_{2}^{2}=0
$$

By the back substitution, we can obtain the final solutions, but they are not real solutions.

$* *$ Sub derivation C2.2.: $z_{3}=0, y_{3}=0$

With $\left(x_{2}-x_{1}\right)=0$ and $z_{3}=0, y_{3}=0$, by solving the original equation we have

$$
\left\{\begin{array}{lll}
x_{1}^{2}=0 ; & x_{2}^{2}=0 ; & x_{3}^{2}=1 \\
y_{1}^{2}=5 / 6 ; & y_{2}^{2}=5 / 6 ; & y_{3}^{2}=0 \\
z_{1}^{2}=1 / 6 ; & z_{2}^{2}=1 / 6 ; & z_{3}^{2}=0
\end{array}\right.
$$

Till now, we have obtain real solutions in the Sub derivation C2.2. and in the Sub derivation $\mathrm{C} 1.1$. It can be found that they are corresponding to the same configurations. A set of real solutions is given as follows:

$$
\left\{\begin{array}{lll}
x_{1}=0 ; & x_{2}=0 ; & x_{3}= \pm 1 \\
y_{1}= \pm \sqrt{5 / 6} ; & y_{2}=\mp \sqrt{5 / 6} ; & y_{3}^{2}=0 \\
z_{1}^{2}= \pm 1 / \sqrt{6} ; & z_{2}^{2}= \pm 1 / \sqrt{6} ; & z_{3}^{2}=0
\end{array}\right.
$$

For example, the following configuration is one of the solutions given above 


$$
\mathbf{J}^{\prime}=\left[\begin{array}{ccc}
0 & \sqrt{5 / 6} & \sqrt{1 / 6} \\
0 & -\sqrt{5 / 6} & \sqrt{1 / 6} \\
1 & 0 & 0 \\
\cos \alpha & 0 & -\sin \alpha \\
\cos \alpha & 0 & \sin \alpha
\end{array}\right]
$$

However, although the solution (58) satisfying the equation (31) from the equation (21), it cannot satisfy the following equation also from (21)

$$
x_{1}^{2}+x_{2}^{2}+x_{3}^{2}+\left(x_{1}+x_{2}+x_{3}\right)^{2} / 2=n / 3
$$

because

$$
x_{1}^{2}+x_{2}^{2}+x_{3}^{2}+\left(x_{1}+x_{2}+x_{3}\right)^{2} / 2=1+1 / 2=3 / 2 \neq 5 / 3
$$

It can be verified that all real solutions of the equation (31) can not satisfy the equation (59) which is one of equation from (21).

\section{(b) Verified by the Mathematica software}

\section{(b1) Codes}






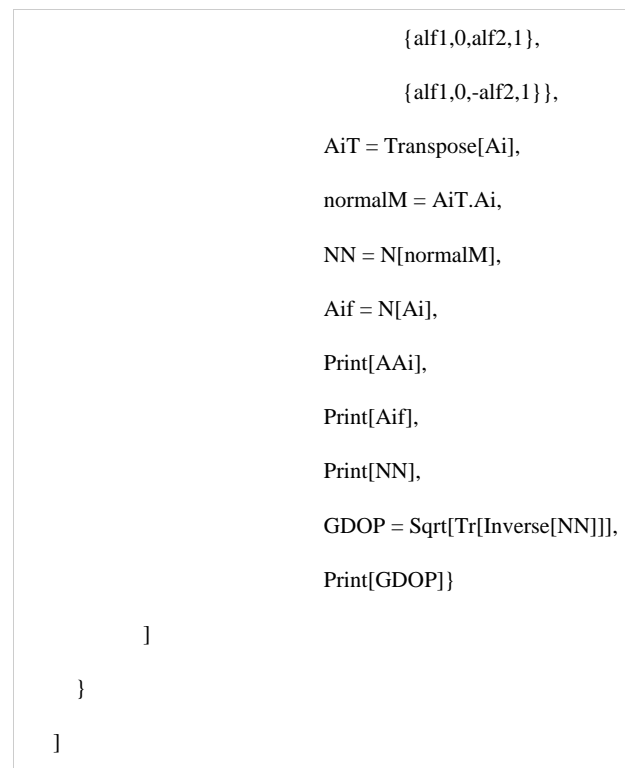

(b2) The different solutions and their GDOPs

Tab.1 Solutions with different geometry from the software

\begin{tabular}{|l|c|}
\hline \multicolumn{1}{|c|}{${\text { Jacobin matrix } \mathbf{J}^{\mathbf{T}}}^{\text {GDOP }}$} \\
\hline$\{-\sqrt{1 / 6}, \sqrt{5 / 6}, 0,-\sqrt{1 / 6},-\sqrt{5 / 6}, 0,-1,0,0\}$ & 1.4522709489247896 \\
\hline$\{\sqrt{1 / 6}, \sqrt{5 / 6}, 0, \sqrt{1 / 6},-\sqrt{5 / 6}, 0,-1,0,0\}$ & 1.428428579164341 \\
\hline$\{\sqrt{1 / 6}, \sqrt{5 / 6}, 0, \sqrt{1 / 6},-\sqrt{5 / 6}, 0,1,0,0\}$ & \\
& 2.441227517915227 \\
\hline
\end{tabular}

Note that the solutions are the same with the Poof above. The results in Tab.1 is calculated with

$$
\cos \alpha=\sqrt{\left(5 / 3-x_{1}^{2}-x_{2}^{2}-x_{3}^{2}\right) / 2}
$$




\section{FINANCIAL SUPPORT}

This work is partly supported by National Science Foundation of China (grant Nos. 41020144004, 41104018, and 41474011). National High-tech R\&D Program (grant Nos.2009AA121405, 2012BAB16B01, 2013AA122501, 2016YFB0501700) and GFZX0301040308-06.

\section{References}

AWANGE, J.L., FUKUDA, Y. and GRAFAREND, E.W., 2004. Exact solution of the nonlinear 7-parameter datum transformation by Groebner basis. Bollettino di geodesia e scienze affini, 63(2): 117-127.

Awange, J.L., Grafarend, E.W., Paláncz, B. and Zaletnyik, P., 2010. Algebraic geodesy and geoinformatics. Springer Berlin Heidelberg, Berlin ; Heidelberg.

Awange, J.L., Grafarend, E.W. and Takemoto, Y.F.S., 2003. Direct Polynomial approach to nonlinear distance (ranging) problems. Earth, Planets and Space(55): 231-241.

Han, T., Wu, H., Lu, X., Du, J. and Zhang, X., 2013. The Mathematical Expectation of GDOP and its Application, China Satellite Navigation Conference (CSNC) 2013 Proceedings. Springer Berlin Heidelberg, pp. 501-521.

Kihara, M. and Okada, T., 1984. A satellite selection method and precision for the global positioning system. NAVIGATION, 31(1): 8-20.

Langley, R.B., 1999. Dilution of precision. GPS world, 10(5): 52-59.

Levanon, N., 2000. Lowest GDOP in 2-D scenarios. IEEE Proceedings-Radar, Sonar and Navigation, 147(3): 149-155.

Massatt, P. and Rudnick, K., 1990. Geometric formulas for dilution of precision calculations. Navigation, 37(4): 379-391.

Phillips, A.H., 1984. Geometrical determination of PDOP. Navigation, 31(4): 329-337.

Sato, M., Fujita, M., Matsumoto, Y., Saito, H., Ishikawa, T., \& Asakura, T., 2013. Improvement of GPS/acoustic seafloor positioning precision through controlling the ship's track line. Journal of Geodesy, 87(9): 825-842.

Sharp, I., Yu, K. and Guo, Y.J., 2009. GDOP analysis for positioning system design. IEEE Transactions on Vehicular Technology, 58(7): 3371-3382.

Sharp, I., Yu, K. and Hedley, M., 2012. On the GDOP and accuracy for indoor positioning. IEEE Transactions on Aerospace and Electronic Systems, 48(3): 2032-2051.

Shim, D.-S. and Yang, C.-K., 2010. Optimal configuration of redundant inertial sensors for navigation and FDI performance. Sensors, 10(7): 6497-6512.

Sukkarieh, S., 2000. Low cost, high integrity, aided inertial navigation systems for autonomous land vehicles, Doctoral dissertation, The University of Sydney.

Teng, Y. and Wang, J., 2014. New characteristics of geometric dilution of precision (GDOP) for multi-GNSS constellations. Journal of Navigation, 67(06): 1018-1028.

Teng, Y., Wang, J. and Huang, Q., 2015. Minimum of Geometric Dilution of Precision (GDOP) for five satellites with dual-GNSS constellations. Advances in Space Research, 56(2): 229-236.

Teunissen, P., 1998. A proof of Nielsen's conjecture on the GPS dilution of precision. Aerospace and Electronic Systems, IEEE Transactions on, 34(2): 693-695.

Xue, S. and Yang, Y., 2015. Positioning configurations with the lowest GDOP and their classification. Journal of Geodesy, 89(1): 49-71. 
Xue, S., Yang, Y. and DANG, Y., 2014a. A closed-form of Newton method for solving over-determined pseudo-distance equations. Journal of Geodesy, 88(5): 441-448.

Xue, S., Yang, Y., Dang, Y. and Chen, W., 2014b. Dynamic positioning configuration and its first-order optimization. Journal of Geodesy, 88(2): 127-143.

Yang, Y., Li, J., Xu, J. and Tang, J., 2011a. Generalised DOPs with consideration of the influence function of signal-in-space errors. Journal of Navigation, 64(S1): S3-S18.

Yang, Y., Li, J., Xu, J., Tang, J., Guo, H., \& He, H., 2011b. Contribution of the compass satellite navigation system to global PNT users. Chinese Science Bulletin, 56(26): 2813-2819.

Yang, Y. and Xu, J., 2016. GNSS receiver autonomous integrity monitoring (RAIM) algorithm based on robust estimation. Geodesy and Geodynamics.

Yarlagadda, R., Ali, I., Al-Dhahir, N. and Hershey, J., 2000. GPS GDOP metric. IEEE Proceedings-Radar, Sonar and Navigation, 147(5): 259-264.

Yuksel, Y., 2011. Design and Analysis of Inertial Navigation Systems with Skew Redundant Inertial Sensors. UCGE Report, 20328.

Zaletnyik, P., Paláncz, B., Awange, J. and Grafarend, E., 2008. Application of Computer Algebra System to Geodesy, Observing our Changing Earth. Springer, Berlin Heidelberg, pp. 803-808.

Zhang, M. and Zhang, J., 2009. A fast satellite selection algorithm: beyond four satellites. IEEE J. Selected Topics Signal Process, 3(5): 740-747.

Zhao, J., Zou, Y., Zhang, H., Wu, Y. and Fang, S., 2015. A new method for absolute datum transfer in seafloor control network measurement. Journal of Marine Science and Technology, 21(2): 216-226. 